\title{
October 2018 Critical Care Case of the Month: A Pain in the Neck
}

\author{
Robert A. Raschke, MD
}

\author{
Critical Care Medicine
}

HonorHealth Scottsdale Osborn Medical Center

Scottsdale, AZ USA

\section{History of Present IIIness}

A 54-year-old man was admitted after he had a decline in mental status. He complained of neck and back pain for one week prior to admission for which he took

acetaminophen. He was seen in the emergency department two days prior to admission and diagnosed with "arthritis" and prescribed oxycodone/acetaminophen and cyclobenzaprine. On the day of admission be became unresponsive and was transported by ambulance to the emergency department where he was intubated for airway protection.

\section{Past Medical History, Social History, Family History}

- Alcoholism

- Hepatitis C

- Esophageal varices

- Family history is noncontributory

\section{Physical Examination}

- Vitals: T $102^{\circ} \mathrm{F}, \mathrm{BP} 150 / 60 \mathrm{~mm} \mathrm{Hg}, \mathrm{P} 114$ beats/min, 20 breaths/min

- Unresponsive

- Dupuytren's contractures, spider angiomata

- $3 / 6$ systolic murmur

- Deep tendon reflexes 3+

- Bilateral Babinski's sign (toes upgoing)

Which of the following are diagnostic considerations at this time?

1. Bacterial endocarditis

2. Hypoglycemia

3. Liver failure

4. 1 and 3

5. All of the above 


\section{Correct! \\ 5. All of the above}

The differential diagnosis for a change in mental status is large. A mnemonic that summarizes the differential and can be used to initiate evaluation AEIOU TIPS (Table 1) (1).

Table 1. Altered mental status mnemonic.

- A - Alcohol/Acidosis

- E - Endocrine/Epilepsy/Electrolytes/Encephalopathy

- I - Infection- meningitis, encephalitis, sepsis, septic shock; pneumonia, urinary tract infection, occult osteomyelitis.

- O-Opiates, Overdose

- U-Uremia/Underdose

- T - Trauma - head injury, blood loss (shock).

- I-Insulin

- P-Poisoning/Psychosis/pharmacology

- S-Stroke/Seizure/syncope

The patient has the potential for many of these. They would include infection with bacterial endocarditis given his loud murmur and fever; liver failure because of his history of prior liver disease and treatment with acetaminophen; and hypoglycemia although he has no particular reason from his history to develop hypoglycemia. Blood was drawn for initial laboratory evaluation and he was given $50 \mathrm{ml}$ of $50 \%$ dextrose intravenously without response. Significant initial laboratory values are given in Table 2 .

Table 2. Initial laboratory values.

- CBC: WBC $26.8 \times 10^{9}$ cells/L (normal $4.3-10.8^{9} \mathrm{cells} / \mathrm{L}$ )

- BUN/creatinine: $93 \mathrm{mg} / \mathrm{dL} / 2.7 \mathrm{mg} / \mathrm{dL}$ (normal 7-20 mg/dL/0.6 to $1.2 \mathrm{mg} / \mathrm{dL}$ )

- Aspartate Aminotransferase (AST): $1222 \mathrm{U} / \mathrm{L}$ (normal 10-40 U/L)

- Ammonia: $122 \mu \mathrm{mol} / \mathrm{L}$ (normal 11 to $32 \mu \mathrm{mol} / \mathrm{L}$ )

- Prothrombin time (PT): 20 seconds (normal 11 to 13.5 seconds)

What should be done next?

1. Begin empiric antibiotics

2. CT of the head

3. Lactulose

4. 1 and 3

5. All of the above 


\section{Correct! \\ 2. $\mathrm{CT} / \mathrm{MRI}$ of the head}

The CT of the head was verbally reported as normal. Consideration was given to meningitis and a lumbar puncture was performed. The laboratory results are given in Table 3.

Table 3. Results from lumbar puncture.

- White blood cells: 139 cells $/ \mathrm{ml}$ (normal $<5$ cells $/ \mathrm{ml}$ ) with $59 \%$ neutrophils

- Glucose: $71 \mathrm{mg} / \mathrm{dL}$ (blood glucose $101 \mathrm{mg} / \mathrm{dL}$, normal 50-80 mg/dL)

- Protein: 114 (normal 15-45 mg/dL)

- Gram stain: negative

What should be done next?

1. After obtaining blood cultures begin empiric antibiotics including coverage for meningitis

2. Review the head CT/MRI of the head with the radiologist

3. Transesophageal echocardiography

4. 1 and 3

5. All of the above 


\section{Correct! \\ 5. All of the above}

The lumbar puncture fluid is not normal with an elevated WBC count and protein but a normal glucose and no organisms seen on Gram stain. Empiric antibiotics of ceftriaxone and vancomycin were begun. A transesophageal echocardiogram did not show evidence of endocarditis. The head CT was reviewed with the radiologist who saw no abnormalities within the cranium. However, given the clinical presentation he suggested an MRI of the head and neck which was performed (Figure 1).

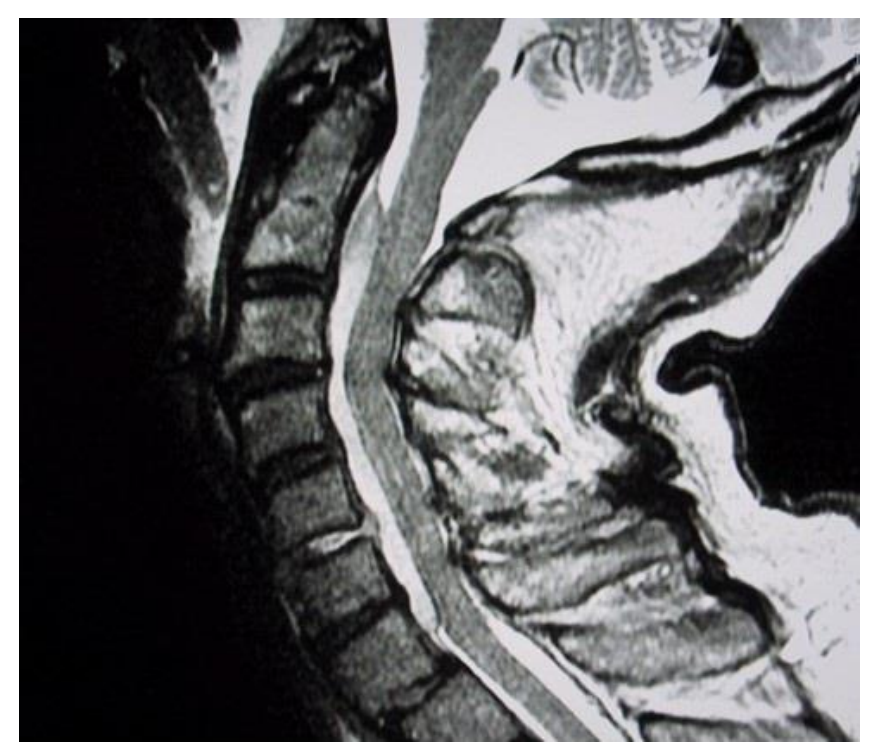

Figure 1. Representative view of MRI of the neck.

Which abnormality (ities) is seen on the MRI of the neck?

1. Boney destruction of $\mathrm{C} 1-\mathrm{C} 4$

2. Epidural abscess

3. Osteomyelitis of $\mathrm{C} 1-\mathrm{C} 4$

4. 1 and 3

5. All of the above 


\section{Correct! \\ 2. Epidural abscess}

The boney structures are intact on the MRI of the neck but there is evidence of an epidural abscess (Figure 2).

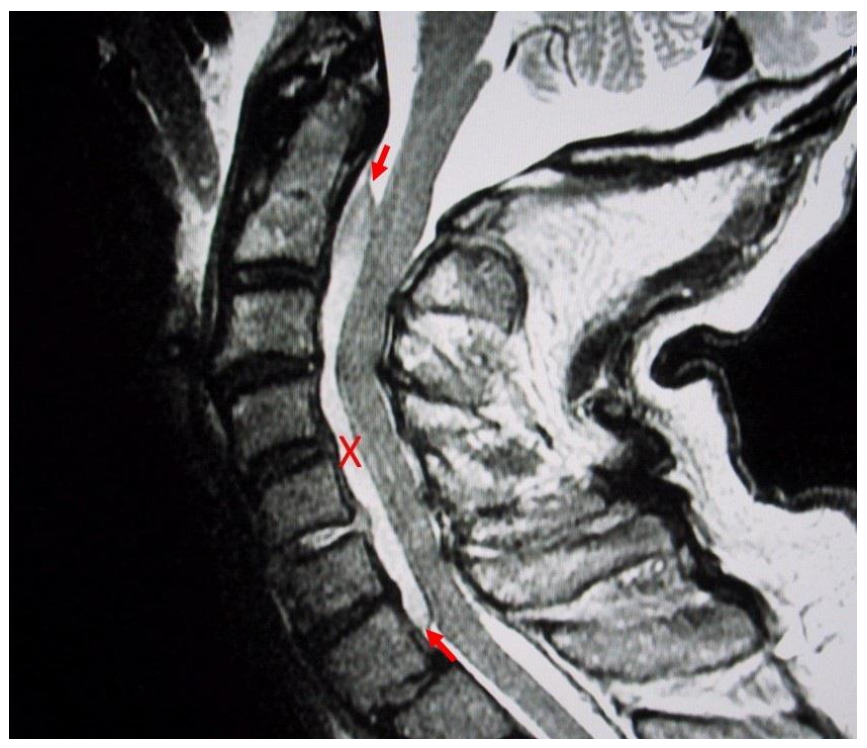

Figure 2. MRI of the neck with epidural abscess highlighted by the red $\mathrm{X}$ between the red arrows.

Which of the following are true regarding epidural abscesses?

1. About $2 / 3$ of cases are due to Staphylococcus aureus

2. An elevated CSF WBC count and protein but a normal glucose and no organisms are typical lumbar puncture findings

3. Treatment consists of medical and surgical therapy

4. 1 and 3

5. All of the above 


\section{Correct! \\ 5. All of the above}

Clinical presentation of paraspinous abscess can be quite variable (2). One half of cases are estimated to be misdiagnosed or have a delayed diagnosis. If untreated, an expanding suppurative infection in the spinal epidural space impinges on the spinal cord, producing sensory symptoms and signs, motor dysfunction, and, ultimately, paralysis and death.

Most recommend a combination of antistaphylococcal antibiotics and surgical decompression. Antibiotic treatment with CT-guided aspiration of the epidural space is increasingly used in patients without neurologic deficits but because of the rarity of the disorder, no randomized trial results are available to guide the clinician.

On day 2 our patient's blood cultures were reported positive for Gram + cocci in clusters and he underwent a $\mathrm{C} 2-\mathrm{C} 7$ laminectomy. By day 3 his strength had recovered and on day 5 the organism in the blood cultures was identified as methicillin-resistance Staphylococcus aureus (MRSA). He was treated with 4 weeks of antibiotic therapy and made a full and uneventful recovery. The source of the infection was never identified.

\section{References}

1. Emergency Medicine/Altered mental status. Wikibooks. Available at: https://en.wikibooks.org/wiki/Emergency Medicine/Altered mental status (accessed 9/7/18).

2. Huff JS. Spinal epidural abscess. Medscape. July 12, 2018. Available at: https://emedicine.medscape.com/article/1165840-overview (accessed 9/7/18). 